\title{
Stage IB Lung Cancer AJCC v8
}

National Cancer Institute

\section{Source}

National Cancer Institute. Stage IB Lung Cancer A/CC v8. NCI Thesaurus. Code C136474.

Stage IB includes: T2a, N0, MO. T2a: Tumor measuring more than $3 \mathrm{~cm}$ but $4 \mathrm{~cm}$ or less

in greatest dimension. NO: No regional lymph node metastases. MO: No distant

metastasis. (AJCC 8th ed.) 